\title{
Palliative sequential chemoradiotherapy for pulmonary sulcus tumor: A case report
}

\author{
MIKI KIKUCHI ${ }^{1}$, TOSHIHIRO OHTANI ${ }^{2}$, TOMOHIRO TAMURA $^{3}$ and HIROAKI SATOH ${ }^{3}$ \\ Divisions of ${ }^{1}$ Nursing, ${ }^{2}$ Pharmacy and ${ }^{3}$ Respiratory Medicine, Mito Medical Center, \\ University of Tsukuba, Mito, Ibaraki 310-0015, Japan
}

Received May 17, 2016; Accepted August 25, 2016

DOI: $10.3892 / \operatorname{mco} .2016 .1026$

\begin{abstract}
Pulmonary sulcus tumors are occasionally encountered in non-small-cell lung cancer (NSCLC) patients. If the patients have a good clinical condition, concurrent chemoradiotherapy is indicated. However, palliative chemotherapy and chemoradiotherapy for patients exhibiting a poor overall condition have not been reported to date. We herein report the case of a 54-year-old female patient with a pulmonary sulcus tumor, who had a poor performance status. The patient received sequential chemoradiotherapy, with no severe complications. There was no local recurrence until the time of death, 8 months after the initiation of therapy. Palliative sequential chemoradiotherapy for pulmonary sulcus NSCLC must be effective and carry low risk of complications. Palliative sequential chemoradiotherapy may be considered in certain patients with pulmonary sulcus NSCLC. Either chemotherapy or radiotherapy is administered first and, if there is no apparent deterioration of the patient's general condition, the next treatment modality is administered.
\end{abstract}

\section{Introduction}

Pulmonary sulcus tumors are a rare type of in lung cancer (1). As the disease is often unresectable, adequate palliative management is crucial. Concurrent chemoradiotherapy for locally advanced non-small-cell lung cancer (NSCLC) patients with a good overall condition has long been a matter for discussion (2). However, to the best of our knowledge, palliative therapy for patients with such tumors who are in a poor overall condition has not been reported to date. In order to increase the awareness of the approach to palliative care of locally advanced NSCLC patients with poor performance status (PS), we herein present the case of a patient with

Correspondence to: Professor Hiroaki Satoh, Division of Respiratory Medicine, Mito Medical Center, University of Tsukuba, Miya-machi 3-2-7, Mito, Ibaraki 310-0015, Japan

E-mail: hirosato@md.tsukuba.ac.jp

Key words: sequential chemoradiotherapy, pulmonary sulcus tumor symptomatic sulcus NSCLC, who was treated with sequential chemoradiotherapy in the palliative setting.

\section{Case report}

A 54-year-old woman was referred to the Mito Medical Center (Mito, Japan) due to complaints suspicious for lung cancer. The patient presented with paralysis in the right upper extremity and severe pain, which required oxycodone administration at $60 \mathrm{mg}$ per day. A computed tomography (CT) scan revealed a pulmonary sulcus tumor in the right lung, with direct invasion of the brachial neural plexus and the thoracic spinal canal, with erosive changes in the thoracic spine and ribs (Fig. 1). The CT scan also showed enlarged ipsilateral mediastinal lymph nodes, an ipsilateral pleural fluid collection, and an enlarged right adrenal gland. Transbronchial biopsy specimens, which were obtained from the tumor through fiberoptic bronchoscopy, revealed lung adenocarcinoma. Genetic analysis of the tumor did not show epidermal growth factor receptor or anaplastic lymphoma kinase mutations. On pretreatment evaluation, the tumor was classified as cT4N2M1b lung adenocarcinoma. The patient had a poor PS (PS 3), poor nutritional status (serum albumin: $3.9 \mathrm{mg} / \mathrm{dl}$ ), and presented with excessive weight loss (body weight, $38.8 \mathrm{~kg}$ ). At first, supportive care was recommended due to the patient's poor clinical condition. However, the patient insisted on multidisciplinary therapy. Therefore, sequential chemoradiotherapy with a palliative intent was selected. The patient received palliative irradiation for the primary lesion (45 Gy in 4.5 weeks), without severe adverse events (AEs) or deterioration of her clinical condition. Two weeks after the palliative irradiation, taking the patient's clinical condition into consideration, palliative chemotherapy with 6 courses of pemetrexed $\left(500 \mathrm{mg} / \mathrm{m}^{2}\right.$ on day $\left.1, \mathrm{q} 28\right)$ was performed. The treatment was successful and uncomplicated. Although the paralysis in right arm did not improve, the pain was effectively controlled without increasing the morphine dose. There was no local recurrence until the time of death from cancer progression, 8 months after the initiation of therapy.

\section{Discussion}

Concurrent chemoradiotherapy is currently considered as the standard therapy for locally advanced NSCLC (3-5) but, due 


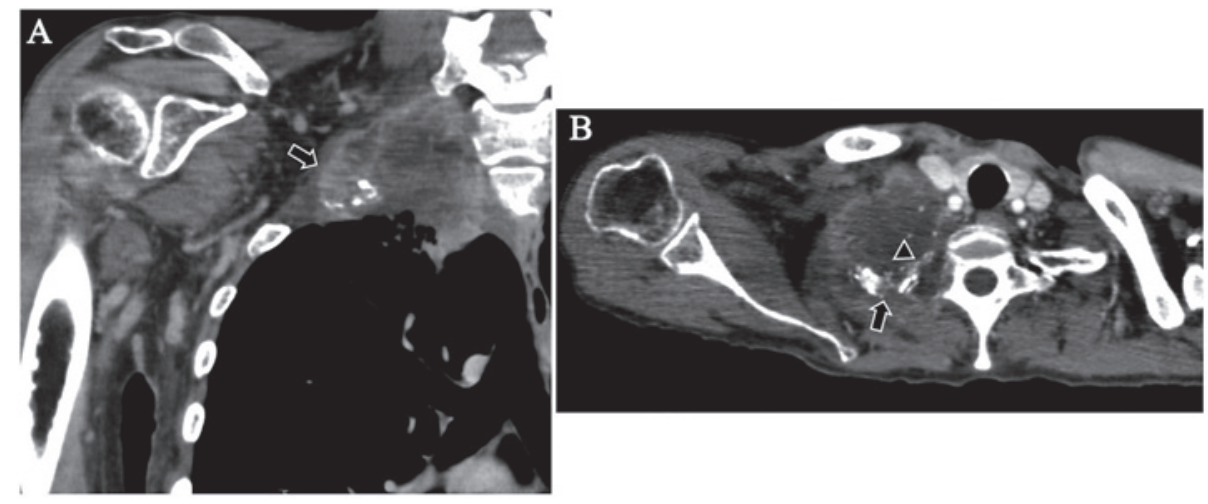

Figure 1. (A) Coronal and (B) axial view of the computed tomography scan showing a pulmonary sulcus tumor in the right lung, with direct invasion of the ribs (arrows) and thoracic spine (arrowhead).

to the severe associated AEs, it is only indicated for NSCLC patients in a good overall condition (3-5). However, studies considering palliative sequential chemoradiotherapy for locally advanced NSCLC have not been reported to date. For patients with metastatic disease, chemotherapy and supportive care are currently considered the standard of care. Palliative radiotherapy for pain control may be effective in some patients. Although only in a limited number of cases, a palliative benefit with thoracic radiotherapy in patients with stage IV NSCLC who have a good PS has rarely been reported $(6,7)$, but chemoradiotherapy for stage IV NSCLC patients has never been shown to provide a survival benefit. As regards chemotherapy for stage IV NSCLC patients with a poor PS, single-agent treatment with docetaxel or pemetrexed may be considered (8). To the best of our knowledge, there have been no reported cases with palliative chemoradiothrapy for stage IV NSCLC patients with a poor PS. Due to the short life expectancy of patients on palliative care, whenever possible, chemoradiotherapy should be non-distressing, effective and short-course. Irradiation may cause dermatitis and/or esophagitis, and chemotherapy may be associated with general fatigue, appetite loss and myelosuppression, whereas hospitalization during the treatment period severely compromises the patient's quality of life.

In addition, the cornerstone of the management for NSCLC with a specific genetic etiology, such as epidermal growth factor receptor mutations, is molecular-targeted therapy (9-11); however, no such specific genetic etiology was identified in the present case. In our patient, standard chemoradiotherapy, either concurrent or sequential, was not indicated, as she had metastatic disease and a poor PS, but the patient requested multidisciplinary treatment. However, any standard concurrent chemoradiotherapy was considered to be intolerable due to her poor condition, as chemotherapy-induced AEs would increase the patient's discomfort. Therefore, palliative sequential chemoradiotherapy was selected and radiotherapy alone was first delivered. As severe AEs did not develop, additional chemotherapy with pemetrexed alone was planned. Pemetrexed was selected as the chemotherapeutic agent, as it is associated with fewer hematological and non-hematological AEs (8); if any severe AEs had developed with this agent, another course of the chemotherapy would not have been indicated.

The patient selection criteria for palliative sequential chemoradiotherapy have not been specifically outlined and must be considered on a case-by-case basis. When considering palliative outcomes in general, poor PS, poor nutritional status and excessive weight loss are associated with a poor outcome. Although chemoradiotherapy is not generally considered in metastatic NSCLC, local control and a survival benefit may be achieved in selected patients. Palliative sequential chemoradiotherapy for pulmonary sulcus NSCLC may be considered as an option, and this treatment would be considered in selected patients. Either chemotherapy or radiotherapy should be administered first and, if there is no apparent deterioration of the patient's general condition, the next treatment modality should be administered.

\section{References}

1. Glassman LR and Hyman K: Pancoast tumor: A modern perspective on an old problem. Curr Opin Pulm Med 19: 340-343, 2013.

2. Peedell $\mathrm{C}$, Dunning $\mathrm{J}$ and Bapusamy A: Is there a standard of care for the radical management of non-small cell lung cancer involving the apical chest wall (Pancoast tumours)? Clin Oncol (R Coll Radiol) 22: 334-346, 2010.

3. Laine AM, Westover KD and Choy H: Radiation therapy as a backbone of treatment of locally advanced non-small cell lung cancer. Semin Oncol 41: 57-68, 2014.

4. Antoni D and Mornex F: Chemoradiotherapy of locally advanced nonsmall cell lung cancer: State of the art and perspectives. Curr Opin Oncol 28: 104-109, 2016.

5. Xu P and Le Pechoux C: Chemoradiotherapy for stage III non-small cell lung cancer: Have we reached the limit? Chin Clin Oncol 4: 45, 2015.

6. Topkan E, Yildirim BA, Guler OC, Parlak C, Pehlivan B and Selek U: Safety and palliative efficacy of single-dose 8-Gy reirradiation for painful local failure in patients with stage IV non-small cell lung cancer previously treated with radical chemoradiation therapy. Int J Radiat Oncol Biol Phys 91: 774-780, 2015.

7. Dandekar VK, Young J, Kiel K, Bonomi P, Fidler MJ, Batus M and Sher DJ: Efficacy and tolerability of palliative split-course thoracic chemoradiotherapy for symptomatic Non-small cell lung cancer. Am J Clin Oncol 38: 605-609, 2015.

8. Sculier JP and Moro-Sibilot D: First- and second-line therapy for advanced nonsmall cell lung cancer. Eur Respir J 33: 915-930, 2009.

9. Bareschino MA, Schettino C, Rossi A, Maione P, Sacco PC, Zeppa R and Gridelli C: Treatment of advanced non small cell lung cancer. J Thorac Dis 3: 122-133, 2011.

10. Thomas A, Liu SV, Subramaniam DS and Giaccone G: Refining the treatment of NSCLC according to histological and molecular subtypes. Nat Rev Clin Oncol 12: 511-526, 2015.

11. Gerber DE, Gandhi L and Costa DB: Management and future directions in non-small cell lung cancer with known activating mutations. Am Soc Clin Oncol Educ Book: e353-e365, 2014. 